\title{
Interference produced by emotional conflict associated with anterior cingulate activation
}

\author{
BRIAN W. HAAS and KAZUFUMI OMURA \\ State University of New York, Stony Brook, New York \\ R. TODD CONSTABLE \\ Yale University School of Medicine, New Haven, Connecticut \\ and \\ TURHAN CANLI \\ State University of New York, Stony Brook, New York
}

\begin{abstract}
The anterior cingulate cortex (ACC) is involved in cognition and emotion. In the classic Stroop task, presentation of stimuli that are in response conflict with one another produces activation in the caudal ACC. In the emotional Stroop task, presentation of emotionally salient stimuli produces activation in the rostral ACC. Presentation of stimuli that are emotionally conflicting should activate the caudal ACC; stimuli that are emotionally salient should activate the rostral ACC. We tested this prediction using functional magnetic resonance imaging while subjects made emotional valence judgments of words overlaid on emotional faces (word-face Stroop task). Emotionally incongruent pairs were responded to more slowly than emotionally congruent pairs. Emotionally incongruent trials were associated with increased activation within the caudal ACC, whereas no ACC activation was found in response to emotional saliency. These results support the conflict-monitoring model of caudal ACC and extend this function to conflict within the domain of emotional stimuli.
\end{abstract}

Emotion and cognition are two fundamental aspects of human mental processes that are believed to interact to account for individual differences in personality (Mischel \& Shoda, 1995), decision making (Damasio, 1994), and aspects of intelligence (Mayer \& Salovey, 1995; Salovey \& Mayer, 1990). One brain region that appears to mediate both affect and cognition is the anterior cingulate cortex (ACC; Compton et al., 2003; Devinsky, Morrell, \& Vogt, 1995; Yamasaki, LaBar, \& McCarthy, 2002). One paradigm that has been successfully exploited to document the role of the ACC in affective and cognitive aspects of information processing has been the Stroop task (Stroop, 1935).

In the classic form of the Stroop task (Stroop, 1935), participants are asked to identify, as quickly and accurately as possible, the color in which a word stimulus is printed. Reaction times (RTs) are longer when the semantic and perceptual dimensions of the stimulus are incongruent (e.g., the word red printed in the color blue) than when they are congruent (the word red printed in the color red) or unrelated (the word house printed in red). Thus, for incongruent trials, the semantic and perceptual dimensions of the stimulus map onto the same construct (color) but

This study was funded by Stony Brook University and by the National Science Foundation, Grant No. BCS-0224221. Correspondence concerning this article should be addressed to B. W. Haas, Department of Psychology, State University of New York, Stony Brook, NY 11794-2500 (e-mail: bhaas@ic.sunysb.edu). lead to different results ("red" for word meaning, "blue" for word appearance). Because this construct is relevant to selecting the correct response, the two dimensions of an incongruent stimulus can be said to be in conflict with each other over the final response output. Neuroimaging studies using the classic Stroop task suggest that this type of conflict is associated with activation of the caudal ACC (Bush et al., 1999; Bush et al., 1998).

A variant of the Stroop task using emotional stimuli has been developed to test some of the tenets of cognitive theories of mood disorders (Beck, 1976; Bower, 1981, 1987; Teasdale, 1983, 1988). As in the classic Stroop, participants are asked to respond to the perceptual dimension of the stimulus (e.g., the color of a printed word like murder), without regard to its semantic dimension. Yet, RTs are longer in response to negative than to neutral stimuli (Derryberry \& Reed, 1998; Gotlib, McLachlan, \& Katz, 1988; Richards, French, Johnson, Naparstek, \& Williams, 1992; Wells \& Matthews, 1994). The reduced RT is unlikely to be due to a conflict between semantic and perceptual dimensions of the stimulus, because each maps onto a different construct (emotional valence vs. color), only one of which is relevant to the task. Rather, reduced RT is thought to represent emotional saliency, which may distract from the task itself (Algom, Chajut, \& Lev, 2004) and has been shown to draw attention away from other stimuli (Pratto \& John, 1991). Neuroimaging studies using the emotional Stroop task suggest that emotionally salient stimuli are associated with activation of the rostral ACC 
(Canli, Amin, Haas, Omura, \& Constable, 2004; Whalen et al., 1998).

Thus, variants of the classic and emotional Stroop tasks can be functionally dissociated within the ACC and are theorized to reflect distinct processes related to response conflict (Botvinick, Braver, Barch, Carter, \& Cohen, 2001) and emotional saliency (Williams, Mathews, \& MacLeod, 1996), respectively. To test these theories empirically, it is necessary to develop a Stroop-like task in which emotionally salient stimuli are presented that have two dimensions that are either in response conflict with each other (i.e., emotionally incongruent, EI) or not (emotionally congruent, EC). We developed a task similar to that of Stenberg, Wiking, and Dahl (1998), which we call the word-face Stroop task. We used this task to test for two predictions. The first was that emotionally salient stimuli in response conflict (i.e., EI), relative to emotionally salient stimuli not in response conflict (i.e., EC), should produce caudal ACC activation. This prediction was based on the fact that both stimulus sets were of comparable emotional salience, but differed in their degree of conflict. We also predicted that emotionally congruent, relative to neutral, stimuli should produce rostral ACC activation. This prediction was based on the fact that both stimulus sets were comparable in their lack of conflict, but differed in their degree of emotional salience.

\section{METHOD}

\section{Subjects}

Thirteen healthy right-handed subjects ( 7 females) were recruited from the campus of Stony Brook University (7) or Yale University (6) (all subjects were scanned with the same scanner at Yale University). The subjects' mean age was 21 years $(S D=2.2$; range, 18-24). They had no history of brain injury, reported no substance abuse within the past 6 months, were not on any mood-altering medication, and had no physical limitations that prohibited them from participating in an fMRI study. The study was conducted with approval from the Institutional Review Boards of both Stony Brook University and Yale University. Informed consent was obtained from all subjects.

\section{Stimuli}

Words were projected approximately across the noses of faces (Figure 1). These word stimuli were selected from a stimulus library, the Affective Norms for English Words set (Bradley \& Lang, 1999). On the basis of normed valence ratings on a scale from 1 to 9 , with 1 indicating the most negative rating and 9 indicating the most positive rating, three sets of 30 stimuli were constructed: negative $(M$, $S D: 1.99, .38)$, positive $(7.79, .49)$, and neutral $(5.18, .35)$. The three sets differed significantly in normed valence ratings $[F(2,87)=$ $1,492.40, p<.0001]$, but not in word length $[F(2,87)=0.47, p=$ $.62]$ or word frequency $[F(2,87)=0.05, p=.95]$. In addition, mean arousal ratings for neutral words $(M=3.70)$ differed significantly from those of positive $[M=5.30 ; t(1,58)=10.34, p<.001]$ and those of negative $[M=5.82 ; t(1,58)=11.51, p<.001]$ words. Positive and negative words also differed significantly from one another in arousal ratings $[t(1,58)=2.39, p=.02]$. Note that this difference was not critical, in that positive and negative words were presented equally throughout the same condition (see Behavioral Procedures and Experimental Design).

Color faces were selected from the MacArthur McDonnell face library (Tottenham, Borscheid, Ellertsen, Marcus, \& Nelson, 2002). Again, three groups of 30 stimuli were created (sad, happy, and neutral). Each group comprised equal numbers (15) of males and females.

\section{Behavioral Procedures and Experimental Design}

The subjects were instructed to make emotional valence (positive, negative, neutral) judgments of words overlaid upon faces. Each subject responded to three types of trials (EI, EC, and neutral, N) (Figure 1), and the subjects were asked to "passively view" during a fixation condition while a "+" was presented in the center of the screen. The EI stimuli were made up of a randomly selected positive or negative word projected onto a randomly selected opposing valence face (e.g., the word party on a sad face); the EC stimuli were again made up of a randomly selected positive or negative word, but were projected onto a randomly selected identical valence face (e.g., the word pride on a happy face); and the $\mathrm{N}$ stimuli were made up of a randomly selected neutral word projected onto a randomly selected neutral face (e.g., the word desk on a neutral face). Trials were presented in alternating 30 -sec affective blocks (EI or EC) and $30-\mathrm{sec}(\mathrm{N})$ or 24-sec (fixation) nonaffective blocks ( $\mathrm{N}$ or fixation period). The order of presentation was counterbalanced across subjects. Each subject was presented with a total of 24 blocks ( 6 per condition) and thus responded to a total of 180 trials ( 60 per condi-

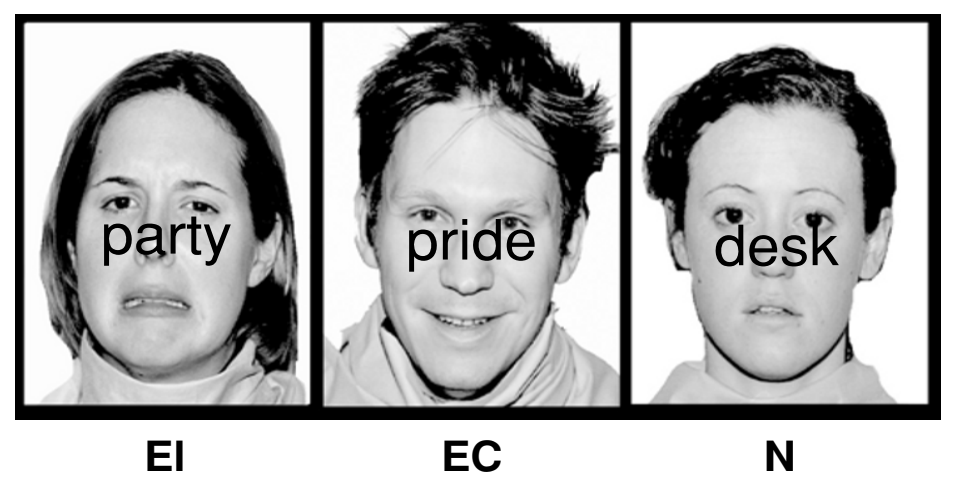

Figure 1. Examples of the three types of stimuli. Emotional incongruent (EI) trials consisted of either a negative or a positive word (e.g.,party) overlaid upon an opposing valence face (e.g., sad). Emotional congruent (EC) trials consisted of either a negative or a positive word (e.g., pride) overlaid upon an identical valence face (e.g., happy). Neutral (N) trials consisted of a neutral word (e.g., desk) overlaid upon a neutral face. All images were presented in color. 
tion). RTs were recorded during the $1,500 \mathrm{msec}$ following the onset of each stimulus.

\section{fMRI Procedures}

Whole-brain imaging data were acquired on a 3T Siemens Trio Scanner. For structural whole-brain images, a 3-D high-resolution spoiled gradient scan and a T1 scan (24 slices, 5-mm thickness; oriented parallel to the line between the anterior and posterior commissures) were conducted. Functional images were acquired using a gradient echo $\mathrm{T} 2 *$-weighted echoplanar imaging (EPI) scan; this was conducted with a flip angle of $80^{\circ}$, repetition time $=1.5 \mathrm{sec}$, echo time $=30 \mathrm{msec}$, and field of view $=220 \times 220 \mathrm{~mm}$ matrix .

Functional data were preprocessed and statistically analyzed using SPM2 (Wellcome Department of Cognitive Neurology). The images were temporally realigned to the middle slice, spatially realigned to the first in the time series, and coregistered to the T1 volume image, which was segmented and normalized to the gray matter template. Spatial transformations derived from normalizing the segmented gray matter were then applied to all functional volumes, which were then spatially smoothed with an 8-mm full-width half-maximum isotropic Gaussian filter.

Fixed effects models (Friston, 1994) were used at the individual subject level of analysis, and random effects models (Holmes \& Friston, 1998) were used for group-level analyses. At the individual level, models were created (general linear model) to represent all conditions (fixation, EI, EC, and N), and all data were then highpass filtered. To determine areas significantly activated in response to emotional conflict, a one-sample $t$ test was performed, subtracting EC from EI; for emotional saliency, the contrast was performed subtracting $\mathrm{N}$ from $\mathrm{EC}$.

For functional analyses restricted to the a priori region of interest (ROI; here, the ACC) an automated method for generating ROI masks based on the Talairach Daemon database (Maldjian, Laurienti, Kraft, $\&$ Burdette, 2003) was used. We applied a significance threshold of $p<.01$ and an extent threshold of 30 voxels (uncorrected) within this a priori ROI, and a significance threshold of $p<.001$ and extent threshold of 40 voxels (uncorrected) in all other regions.

\section{RESULTS}

\section{Reaction Time}

The mean RTs recorded in each of the three task conditions are presented in Figure 2. A $3 \times 1$ ANOVA revealed that subjects differed significantly in RTs among all of the task conditions $[F(2,36)=24.47, p<.001]$. As is apparent in Figure 2, subjects responded considerably slower (57.10-msec difference) to EI parings relative to EC parings $[F(1,24)=10.38, p<.005]$. Subjects also responded significantly slower (57.52-msec difference) to EC pairings relative to the $\mathrm{N}$ pairings $[F(1,24)=13.44$, $p<.005]$. The error rates for each of the conditions were $7.1 \%(\mathrm{EI}), 4.9 \%(\mathrm{EC})$, and $2.9 \%(\mathrm{~N})$. An analysis of these error rates from the three conditions revealed no significant difference $[F(2,36)=2.35, p=.11]$.

\section{ACC Activation As a Function of Emotional Conflict}

Figure 3 displays activation within the ACC as revealed by the contrast of the EI condition relative to the EC condition. In agreement with the conflict monitoring model, emotional incongruency resulted in significant activation within the caudal ACC (MNI coord: 4, 32, 26; 37 voxels; $t=3.15$, $p<.01)$. On the other hand, emotional conflict did not result in any significant activation within the rostral ACC.

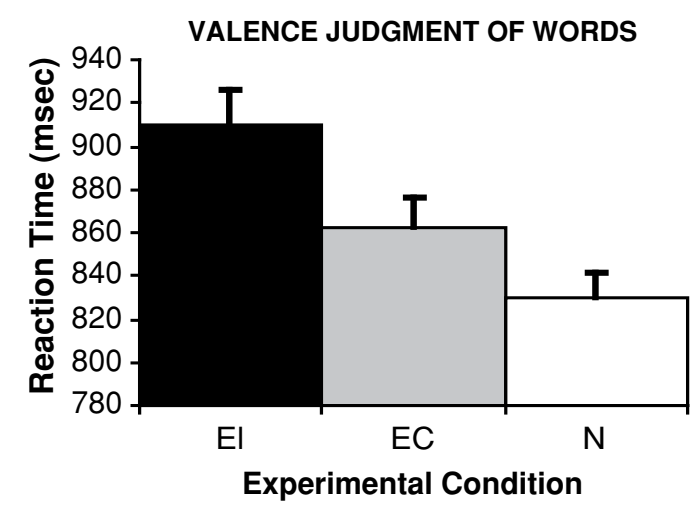

Figure 2. Mean reaction time for responses to emotional words overlaid upon faces in each experimental condition. The respective difference between the emotionally incongruent (EI) condition and the emotionally congruent $(\mathrm{EC})$ condition reveals an effect of emotional conflict. N, neutral. Error bars represent one standard error of the mean.

\section{ACC Activation as a Function of Emotional Saliency}

Contrary to our predictions, no statistically significant clusters were found localized in the rostral ACC when comparing EC trials relative to $\mathrm{N}$ trials. Extending our search area slightly more superior revealed two significant clusters likely localized on the border of the caudal ACC and the Supplementary Motor Area (SMA) (MNI coord: $4,4,50 ; 104$ voxels; $t=5.48, p<.001$; and $-2,6,48$; 109 voxels; $t=4.97, p<.001)$.

\section{Activation Beyond the ACC}

No clusters were found in any other regions when EI trials were compared with EC trials (emotional conflict) at our chosen significance threshold $(p<.001$, extent threshold of 40 voxels). Interestingly, a comparison of the EC trials and $\mathrm{N}$ trials (emotional saliency) did reveal three significant clusters. These clusters were localized on the right precentral gyrus (MNI coord: $30,-8,46$; 760 voxels; $t=6.91, p<.001$ ), the right thalamus (MNI coord: $16,-16,8 ; 204$ voxels; $t=6.14, p<.001$ ), and the left inferior parietal lobule (MNI coord: $-24,-44,50 ; 44$ voxels; $t=4.55, p<.001)$. In a post hoc analysis, we used a reduced threshold $(p<.05$ uncorrected, extent threshold of 10 voxels) in order to investigate amygdala activation patterns in response to task manipulations. This analysis indicated that the left amygdala was activated when EC trials were compared with $\mathrm{N}$ trials (MNI coord: $-20,-10,-10 ; 13$ voxels; $t=2.42, p<.05)$ but not when EI trials were compared with EC trials. (Note that this activation should be viewed with caution, owing to the relatively low significance threshold.)

\section{DISCUSSION}

Imaging data from classic and emotional Stroop tasks have suggested that the ACC has functionally dissociable regions that are sensitive to response conflict caudally 


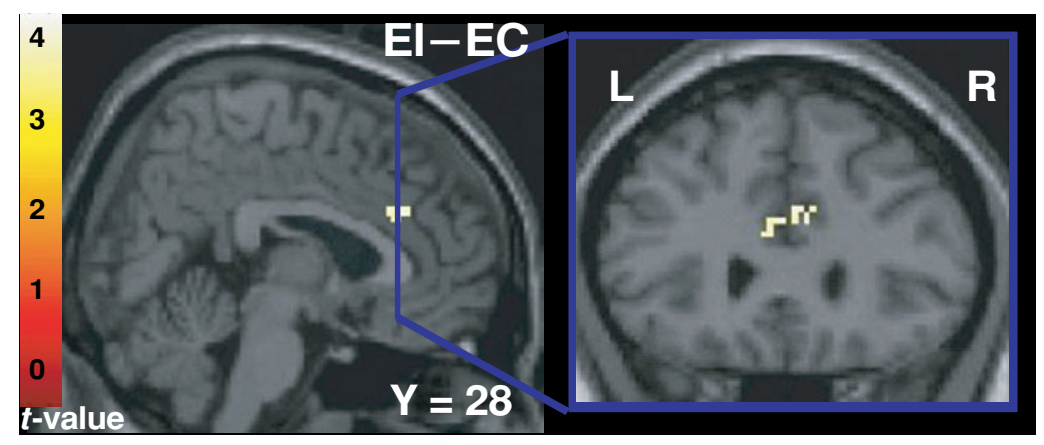

Figure 3. ACC activation in response to emotional conflict: Sagittal $(x=4)$ and coronal $(y=25)$ views displaying increased caudal ACC activation in response to emotional conflict (EI-EC). This contrast revealed a significantly activated cluster localized in the right caudal ACC $(4,32,26 ; 37$ voxels; $t=3.15$, $p<.01)$. No significant activation was found in the rostral ACC.

(Botvinick et al., 2001) and emotional saliency rostrally (Whalen et al., 1998). We predicted that two conditions matched for emotional saliency but that differed in emotional response conflict should therefore generate significant activation in the caudal ACC, whereas an emotionally salient condition relative to a neutral condition should activate the rostral ACC. To test this prediction, we devised a neuroimaging study to use a Stroop-based task, the wordface Stroop task (Stenberg et al., 1998). In support of our prediction, we found that EI stimuli produced greater activation in the caudal ACC than did EC stimuli. The difference in neural activation was associated with a behavioral measure of interference: Subjects' RTs were slower on EI trials than on EC trials. This observation is consistent with a behavioral study that reported longer RTs for positive than for negative words, when they were paired with negative facial expressions (Stenberg et al., 1998).

Contrary to our predictions, we did not find any activation within the rostral ACC in response to emotional saliency. Previous research has found rostral ACC activation when comparing emotionally salient and neutral stimuli during the emotional Stroop task (Canli et al., 2004; Whalen et al., 1998). The current word-face Stroop task is similar to the emotional Stroop task in this respect, but it is also quite different in a number of ways. For example, the emotional Stroop task involves dealing with distracting emotional information (semantic) when one is processing neutral taskrelevant (color) information, whereas the word-face Stroop task involves processing emotionally salient information on both the distracting (face) dimension and the task-relevant (word) dimension. Differences also exist with respect to task instructions. Subjects are asked to make a color judgment during the emotional Stroop task, but they are asked to make a valence judgment during the word-face Stroop task. The contrast of EC relative to $\mathrm{N}$ did reveal activation localized on the border of the SMA and caudal ACC. This finding may be rooted in differences in response selection between the two conditions. During the EC condition, two correct responses (positive and negative) are available, whereas in the $\mathrm{N}$ condition, there is only one (neutral). In- deed, others have found similar clusters of activation when comparing trials with greater response selection relative to trials with less (Lau, Rogers, \& Passingham, 2006).

In the present study, the incongruent condition produced slower behavioral responses than did the congruent condition, providing behavioral evidence for greater interference. However, the behavioral response difference between trial conditions also confounds brain activation associated with the EI condition. It is unlikely, however, that the observed caudal ACC activation is simply related to a motor response, because activation was not correlated with RT (data not shown).

In addition to the classic Stroop, other paradigms have been developed for studying neural responses to conflicting information, such as the flanker (Barch et al., 2001; Bunge, Hazeltine, Scanlon, Rosen, \& Gabrieli, 2002) and go/no-go (Braver, Barch, Gray, Molfese, \& Snyder, 2001; Durston, Thomas, Worden, Yang, \& Casey, 2002) tasks.

On the basis of this work, Botvinick and colleagues have proposed that the caudal ACC is involved in signaling the occurrence of conflicts in information processing (e.g., Botvinick, Cohen, \& Carter, 2004). The authors proposed that the caudal ACC acts as a "conflict monitoring" unit. Our finding of greater caudal ACC activation during EI trials is consistent with this model and extends it to conditions of emotional conflict.

At least two mechanisms may be associated with caudal ACC activation during EI trials. One mechanism may involve attentional processes related to the suppression of conflict-generating information. If there is conflict across the semantic and perceptual stimulus dimensions, attentional resources may be engaged to disregard or suppress perceptual information, because the task requires only a response to semantic information. An alternative mechanism may involve executive processes that compare projected response outcomes against the rules of the task, followed by the selection of the response that is consistent with task demands and/or rejection of the response that is inconsistent with task demands. However, future studies will be needed to address which of these mechanisms, or 
what additional alternative models, can best explain caudal ACC activation during emotional conflict.

The conclusions that can be drawn from our analyses are limited to some extent by our choice of stimuli and task instructions. For example, the present task assessed activation only in response to emotional conflict manipulated by perceptual stimuli. Other mechanisms may be involved in the processing of emotional conflict between internal and external mood states. Furthermore, we did not include a condition for implicit processing of emotion (such as in the emotional Stroop task). The inclusion of this type of condition may clarify the lack of activation found in the rostral ACC.

In summary, the present study explicitly tested and affirmed a model that ascribed sensitivity to conflict to the caudal ACC. By using the emotional word-face Stroop task and balancing emotional saliency in the congruent and incongruent conditions, we extended the presumed role of the caudal ACC to the processing of conflicting information in an emotional context.

\section{REFERENCES}

Algom, D., Chajut, E., \& Lev, S. (2004). A rational look at the emotional Stroop phenomenon: A generic slowdown, not a Stroop effect. Journal of Experimental Psychology: General, 133, 323-338.

Barch, D. M., Braver, T. S., AKbudak, E., Conturo, T., Ollinger, J., \& SNYDER, A. (2001). Anterior cingulate cortex and response conflict: Effects of response modality and processing domain. Cerebral Cortex, 11, 837-848.

BECK, A. T. (1976). Cognitive therapy and the emotional disorders. New York: International Universities Press.

Botvinick, M. M., Braver, T. S., Barch, D. M., Carter, C. S., \& Cohen, J. D. (2001). Conflict monitoring and cognitive control. Psychological Review, 108, 624-652.

Botvinick, M. M., Cohen, J. D., \& Carter, C. S. (2004). Conflict monitoring and anterior cingulate cortex: An update. Trends in Cognitive Sciences, 8, 539-546.

Bower, G. H. (1981). Mood and memory. American Psychologist, 36, 129-148.

Bower, G. H. (1987). Commentary on mood and memory. Behaviour Research \& Therapy, 25, 443-455.

Bradley, M. M., \& Lang, P. J. (1999). Affective norms for English words $(A N E W)$ : Stimuli, instruction manual and affective ratings (Technical Report C-1). Gainesville: University of Florida, Center for the Research in Psychophysiology.

Braver, T. S., Barch, D. M., Gray, J. R., Molfese, D. L., \& Snyder, A. (2001). Anterior cingulate cortex and response conflict: Effects of frequency, inhibition and errors. Cerebral Cortex, 11, 825-836.

Bunge, S. A., Hazeltine, E., Scanlon, M. D., Rosen, A. C., \& GaBRIELI, J. D. E. (2002). Dissociable contributions of prefrontal and parietal cortices to response selection. NeuroImage, 17, 1562-1571.

Bush, G., Frazier, J. A., Rauch, S. L., Seidman, L. J., Whalen, P. J., JENIKE, M. A., ET AL. (1999). Anterior cingulate cortex dysfunction in attention-deficit/hyperactivity disorder revealed by fMRI and the counting Stroop. Biological Psychiatry, 45, 1542-1552.

Bush, G., Whalen, P. J., Rosen, B. R., Jenike, M. A., McInerney, S. C., \& RAUCH, S. L. (1998). The counting Stroop: An interference task specialized for functional neuroimaging: Validation study with functional MRI. Human Brain Mapping, 6, 270-282.

Canli, T., Amin, Z., HaAs, B., Omura, K., \& Constable, R. T. (2004). A double dissociation between mood states and personality traits in the anterior cingulate. Behavioral Neuroscience, 118, 897-904.

Compton, R. J, Banich, M. T., Mohanty, A., Milham, M. P., HerRington, J., Miller, G. A., ET AL. (2003). Paying attention to emotion: An fMRI investigation of cognitive and emotional Stroop tasks. Cognitive, Affective, \& Behavioral Neuroscience, 3, 81-96.

Damasio, A. R. (1994). Descartes' error: Emotion, reason, and the human brain. New York: Putnam.
DERryberRy, D., \& ReED, M. A. (1998). Anxiety and attentional focusing: Trait, state and hemispheric influences. Personality \& Individual Differences, 25, 745-761.

Devinsky, O., Morrell, M. J., \& Vogt, B. A. (1995). Contributions of anterior cingulate cortex to behaviour. Brain, 118, 279-306.

Durston, S., Thomas, K. M., Worden, M. S., Yang, Y., \& Casey, B. J. (2002). The effect of preceding context on inhibition: An event-related fMRI study. NeuroImage, 16, 449-453.

FrISTON, K. J. (1994). Statistical parametric mapping. In R. W. Thatcher, M. Hallett, T. Zeffiro, E. R. John, \& M. Huerta (Eds.), Functional neuroimaging: Technical foundations (pp. 79-93). San Diego: Academic Press.

Gotlib, I. H., Mclachlan, A. L., \& Katz, A. N. (1988). Biases in visual attention in depressed and nondepressed individuals. Cognition \& Emotion, 2, 185-200.

Holmes, A. P., \& Friston, K. J. (1998). Generalisability, random effects and population inference. NeuroImage, 7, S754.

Lau, H., Rogers, R. D., \& Passingham, R. E. (2006). Dissociating response selection and conflict in the medial frontal surface. NeuroImage, 29, 446-451.

Maldjian, J. A., Laurienti, P. J., Kraft, R. A., \& Burdette, J. H. (2003). An automated method for neuroanatomic and cytoarchitectonic atlasbased interrogation of fMRI data sets. Neurolmage, 19, 1233-1239.

Mayer, J. D., \& Salovey, P. (1995). Emotional intelligence and the construction and regulation of feelings. Applied \& Preventive Psychology, 4, 197-208.

Mischel, W., \& SHOdA, Y. (1995). A cognitive-affective system theory of personality: Reconceptualizing situations, dispositions, dynamics, and invariance in personality structure. Psychological Review, 102, 246-268.

Pratto, F., \& John, O. P. (1991). Automatic vigilance: The attentiongrabbing power of negative social information, Journal of Personality \& Social Psychology, 61, 380-391.

Richards, A., French, C. C., Johnson, W., NAPARStek, J., \& Williams, J. (1992). Effects of mood manipulation and anxiety on performance of an emotional Stroop task. British Journal of Psychology, 83, 479-491.

SAlovey, P., \& MAYeR, J. D. (1990). Emotional intelligence. Imagination, Cognition, \& Personality, 9, 185-211.

Stenberg, G., Wiking, S., \& Dahl, M. (1998). Judging words at face value: Interference in a word processing task reveals automatic processing of affective facial expressions. Cognition \& Emotion, 12, 755-782.

STRoop, J. R. (1935). Studies of interference in serial verbal reactions. Journal of Experimental Psychology, 18, 643-662.

Teasdale, J. D. (1983). Negative thinking in depression: Cause, effect, or reciprocal relationship? Advances in Behavior Research \& Therapy, 5, 3-25.

Teasdale, J. D. (1988). Cognitive vulnerability to persistent depression: An investigation of two hypotheses. British Journal of Clinical Psychology, 26, 113-126.

Tottenham, N., Borscheid, A., Ellertsen, K., Marcus, D. J., \& Nelson, D. A. (2002, April). Categorization of facial expressions in children and adults: Establishing a larger stimulus set. Poster presented at the annual meeting of the Cognitive Neuroscience Society, San Francisco.

Wells, A., \& Matthews, G. (1994). Attention and emotion: A clinical perspective. Hillsdale, NJ: Erlbaum.

Whalen, P. J., Bush, G., McNally, R. J., Wilhelm, S., McInerney, S. C., JeniKe, M. A., \& RaUCH, S. L. (1998). The emotional counting Stroop paradigm: A functional magnetic resonance imaging probe of the anterior cingulate affective division. Biological Psychiatry, 44, 1219-1228.

Williams, J. M. G., Mathews, A., \& MacLeod, C. (1996). The emotional Stroop task and psychopathology. Psychological Bulletin, 120, 3-24.

Yamasaki, H., LaBar, K. S., \& McCarthy, G. (2002). Dissociable prefrontal brain systems for attention and emotion. Proceedings of the National Academy of Sciences, 99, 11447-11451.

(Manuscript received December 21, 2004; revision accepted for publication October 30, 2005.) 